\title{
Síndrome hemolítico urémico en una mujer adulta mayor: reporte de caso y revisión de la literatura
}

\section{Hemolytic uremic syndrome in an older adult woman: case report and literature review}

\author{
Diana María Moya-Acuña', Carlos Rosero-Arellano²
}

\begin{abstract}
Resumen. El síndrome hemolítico urémico (SHU) típico en adultos es una patología infrecuente. En la literatura se encuentran pocos reportes, y se ha documentado principalmente en la población pediátrica. Esta entidad se caracteriza por ser una microangiopatía trombótica (MAT) que compromete de manera característica los riñones. Es causada usualmente por la infección por Escherichia coli productora de toxina Shiga (STEC), especificamente el serotipo 0157:H7. En Colombia no existen casos reportados sobre esta condición en adultos, lo cual llama la atención, pero puede deberse en parte a las dificultades en su diagnóstico, al no tenerse fácil acceso a algunas de las pruebas que orientan hacia esta enfermedad y confirman el diagnóstico. Se reporta el caso de una mujer adulta mayor colombiana, quien consultó por deposiciones diarreicas y hematoquecia, con el posterior desarrollo de trombocitopenia severa, lesión renal aguda, y evidencia de equinocitos y esquistocitos en extendido de sangre periférica, lo que llevó a sospechar una MAT. Se le solicitó FilmArray ${ }^{\circledR}$ gastrointestinal, el cual fue positivo para STEC, confirmando así el diagnóstico de un SHU típico. Se presenta también una breve revisión del tema de una entidad que requiere un diagnóstico temprano y certero que permita brindar un tratamiento eficaz y oportuno.
\end{abstract}

Palabras clave: microangiopatías trombóticas, síndrome hemolítico urémico, Escherichia coli, toxina Shiga, diarrea, trombocitopenia, anemia hemolítica, lesión renal aguda.

Abstract. The classic or typical hemolytic uremic syndrome (HUS) in adults is a rare disease. Few reports are found in the literature, and it has mainly been documented

\footnotetext{
1 Médica Interna, Universidad de la Sabana. Cundinamarca, Colombia.

${ }^{2}$ Médico, Especialista en Medicina Interna, Clínica Universidad de la Sabana. Docente Clínico, Facultad de Medicina, Universidad de la Sabana. Cundinamarca, Colombia. E-mail: chra31@gmail.com.

Conflicto de interés: los autores declaran que no tienen conflicto de interés.

Medicina \& Laboratorio 2021;25:535-546. https://doi.org/10.36384/01232576.438.

Recibido el 8 de julio de 2020; aceptado 19 de diciembre de 2020. Editora Médica Colombiana S.A., 2021
} 
in the pediatric population. This condition is a form of thrombotic microangiopathy (TMA), which characteristically compromises the kidneys. It is mainly caused by infection with Shiga toxin-producing Escherichia coli (STEC), specifically the O157:H7 serotype. In Colombia there are no reports on this condition in adults, and may be due in part to difficulties in its diagnosis, as there is not easy access to some of the tests that guide towards this condition and confirm the diagnosis. The case of an elderly Colombian woman is reported, who presented diarrhea and hematochezia, and subsequently developed severe thrombocytopenia and acute kidney injury, with evidence of echinocytes and schistocytes in peripheral blood smears, which led to suspect TMA. A gastrointestinal FilmArray ${ }^{\mathrm{TM}}$ was ordered, which was positive for STEC, thus confirming the diagnosis of a typical HUS. A brief literature review is also presented, which covers general concepts of a condition that requires an early and accurate diagnosis in order to provide an effective and timely treatment.

Keywords: thrombotic microangiopathies, hemolytic uremic syndrome, Escherichia coli, Shiga toxin, diarrhea, thrombocytopenia, hemolytic anemia, acute kidney injury.

\section{Introducción}

El síndrome hemolítico urémico ( $\mathrm{SHU}$ ) típico, junto con el SHU atípico y la púrpura trombocitopénica trombótica, forman parte de las microangiopatías trombóticas (MAT). Estas entidades, aunque raras, se caracterizan por presentar anemia hemolítica microangiopática, trombocitopenia y trombos plaquetarios que conducen a oclusión microvascular en órganos como riñón y cerebro. Por otra parte, se diferencian por los mecanismos fisiopatológicos; el SHU típico causado como resultado de una infección bacteriana, el SHU atípico asociado a alteraciones mediadas por el complemento, en tanto que la púrpura trombocitopénica trombótica es causada por una deficiencia severa de la enzima ADAMTS13 [1].

Específicamente, los pacientes que cursan con SHU típico presentan la tríada de anemia hemolítica microangiopática, trombocitopenia y falla renal aguda. $\mathrm{Su}$ incidencia global es de 1 a 2 casos por 100.000 habitantes por año [2], y se presenta por lo general en la población pediátrica, siendo raro en los adultos. La principal etiología del SHU típico es la bacteria Escherichia coli, serotipo O157:H7, productora de toxina Shiga STEC (del inglés, Shiga Toxin-like Producing Escherichia Coli), la cual representa más del $90 \%$ de los casos en Estados Unidos y más del $70 \%$ de los casos en Latinoamérica [2,3]. Dentro de su fisiopatología, se describe el daño endotelial que causa la toxina Shiga al ser liberada por lisis bacteriana, la cual forma complejos que llevan a la muerte de las células endoteliales, y aunque los riñones son el principal blanco, pueden desarrollarse complicaciones extrarrenales [4].

Debido a que en la literatura existen pocos reportes de esta patología en adultos, y en Colombia no se han documentado casos similares, se presenta el siguiente caso clínico y se hace una breve revisión del tema, que abarca los conceptos generales, como la clínica, el diagnóstico y el tratamiento de una entidad que requiere un diagnóstico temprano y certero. 


\section{Caso clínico}

Paciente femenina de 83 años quien ingresó a urgencias el 24 de marzo de 2020, por presencia de dolor abdominal tipo cólico de tres días de evolución, quien durante el día previo a la consulta presentó múltiples episodios de melenas y hematoquecia sin otra sintomatología asociada, con antecedente de vitiligo e hipertensión arterial crónica tratada con olmesartán/ amlodipino, sin antecedentes de viajes recientes o sospecha de consumo de algún alimento contaminado.

La paciente se encontró clínicamente estable al ingreso, con tacto rectal positivo para sangrado, hemoglobina normal (tabla 1), con discreta leucocitosis con neutrofilia (90\%), función renal preservada, proteína $C$ reactiva mínimamente elevada, coproscópico con leucocitos y hematíes, sangre oculta positiva, sin evidencia de microorganismos. Al siguiente día se le realizó colonoscopia, la cual reportó proctocolitis ulcerativa versus infecciosa, por lo que el servicio de Gastroenterología indicó ciclo corto de corticoide y mesalazina oral, en tanto que el estudio de endoscopia reportó gastritis crónica antral sin evidencia de sangrado.

Fue valorada por el servicio de Medicina Interna, donde se consideró que por su grupo etario no se podía establecer una proctocolitis ulcerativa como primer diagnóstico, y debido a la presencia de leucocitosis con neutrofilia, se concluyó que la paciente cursaba con proctocolitis de origen infeccioso, por lo que se adicionó tratamiento antibiótico con metronidazol y ciprofloxacina, y se suspendieron los esteroides sistémicos; sin embargo, la paciente persistió con deposiciones diarreicas y hematoquecia.

El 27 de marzo se documentó descenso de plaquetas con respecto al día anterior (tabla 1, figura 1A) y aumento de azoados (tabla 1), con discreto descen-

\begin{tabular}{|c|c|c|c|c|c|c|c|c|c|}
\hline Prueba & $\begin{array}{c}\text { Ingreso } \\
24 / 03\end{array}$ & $27 / 03$ & $28 / 03$ & $06 / 04$ & $17 / 04$ & $23 / 04$ & $29 / 04$ & $\begin{array}{c}\text { Egreso } \\
05 / 06\end{array}$ & $\begin{array}{c}\text { Valores } \\
\text { normales }\end{array}$ \\
\hline $\begin{array}{l}\text { Leucocitos } \\
\left(10^{3} / \mathrm{uL}\right)\end{array}$ & 10,5 & 13,04 & 8,49 & 18,58 & 6,42 & 23,91 & 16,11 & 11,55 & $5-10$ \\
\hline $\begin{array}{l}\text { Neutrófilos } \\
\left(10^{3} / u L\right)\end{array}$ & 9,48 & 11,3 & 5,96 & 16,8 & 4,56 & 22,85 & 6,57 & 8,08 & $2-7,15$ \\
\hline $\begin{array}{l}\text { Hemoglobina } \\
\text { (g/dL) }\end{array}$ & 14,9 & 13,8 & 12,3 & 6,4 & 8,7 & 9,9 & 9,8 & 9 & $12-16$ \\
\hline $\begin{array}{l}\text { Plaquetas } \\
\left(10^{3} / \mathrm{uL}\right)\end{array}$ & 285 & 121 & 15 & 102 & 244 & 391 & 349 & 415 & $150-450$ \\
\hline $\begin{array}{l}\text { Creatinina } \\
(\mathrm{mg} / \mathrm{dL})\end{array}$ & 0,76 & 1,31 & 1,4 & 2,21 & 4,2 & 1,92 & 1,41 & 1,12 & $0,51-0,95$ \\
\hline $\begin{array}{l}\text { Nitrógeno } \\
\text { ureico } \\
(\mathrm{mg} / \mathrm{dL})\end{array}$ & 20 & 28,9 & 43 & 40,7 & 82,4 & 39,6 & 19,7 & 17,2 & $8-23$ \\
\hline $\begin{array}{l}\text { Proteína C } \\
\text { reactiva } \\
(\mathrm{mg} / \mathrm{dL})\end{array}$ & 8,53 & 83,7 & 31,55 & 57,8 & 19 & 34,38 & - & 8,3 & $0,3-5$ \\
\hline
\end{tabular}


so de la hemoglobina (tabla 1, figura 1B). Mientras se esperaba el resultado de la biopsia, se decidió reiniciar el tratamiento corticoide; la biopsia posteriormente reportó colitis isquémica. En el extendido de sangre periférica se evidenciaron equinocitos y esquistocitos sugestivos de hemólisis microangiopática, y plaquetas con morfología normal. Se le realizaron otras pruebas; entre ellas, serología para $\mathrm{VIH}$, toxinas A y B para Clostridium difficile, y glutamato deshidrogenasa (GDH), todas negativas. Los azoados se encontraron en ascenso, con creatinina al ingreso de $0,76 \mathrm{mg} / \mathrm{dL}$, la cual continuó aumentando (figura 1C). Se le realizó ecografía de abdomen en la que se evidenció bazo de tamaño disminuido, con probable infarto esplénico. Posteriormente, la paciente presentó trombocitopenia severa con recuento de plaquetas de $15.000 / u L$, por lo que se indicó transfusión de plaquetas. Por sospecha de púrpura trombocitopénica trombótica versus síndrome hemolítico urémico, se solicitó, posteriormente, el panel gastrointestinal mediante el sistema FilmArray ${ }^{\circledR}$, en materia fecal [5].

Debido al deterioro progresivo, la paciente requirió traslado a $\mathrm{UCl}$, donde se recibió el reporte del FilmArray ${ }^{\circledR}$ positivo para STEC, que confirmó el diagnóstico de SHU típico, y por parte del Comité de Infecciones se suspendió el tratamiento antibiótico. Por la evolución clínica inadecuada de la paciente, fue valorada por Nefrología y se indicó plasmaféresis [6]. La alteración de la función renal persistió con niveles de creatinina de $2,21 \mathrm{mg} / \mathrm{dL}$, que continuaron en aumento hasta llegar a 4,2 $\mathrm{mg} / \mathrm{dL}$ (tabla 1, figura 1C), por lo que se le inició terapia de reemplazo renal. Durante su estancia en $\mathrm{UCl}$ presentó complicaciones propias del paciente hospitalizado, tales como falla ventilatoria, neumonía nosocomial, colitis por

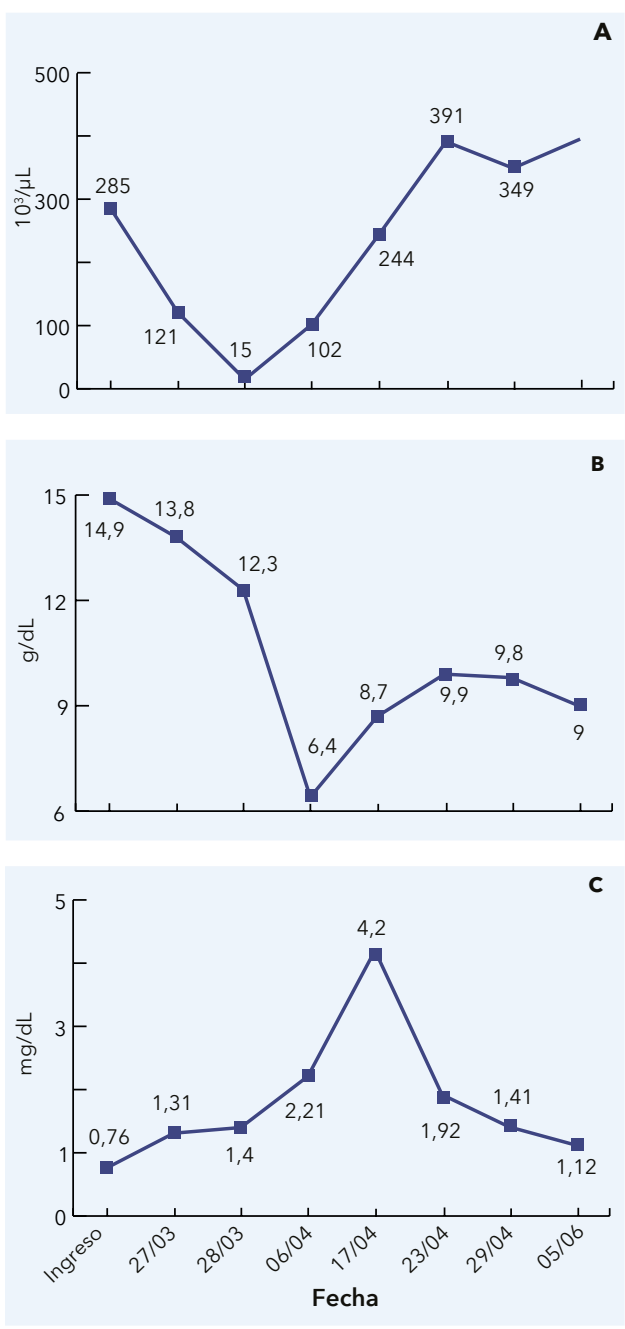

Figura 1. (A) Variación en el número de plaquetas, (B) hemoglobina y (C) creatinina durante la hospitalización.

Clostridium difficile y delirium, las cuales fueron superadas con el tratamiento adecuado. La paciente no presentó nuevos episodios de deposiciones diarreicas.

Otras pruebas realizadas durante la hospitalización se describen en la tabla 2, entre la cuales se observó discreta elevación de las bilirrubinas directa e indirecta, aumento importante de la LDH, y haptoglobina disminuida, que 
se correlacionaron con la anemia de tipo hemolítico.

Finalmente, debido a que la paciente presentó mejoría de la función renal, reflejada por la disminución de los azoados (tabla 1, figura 1C), con suspensión de la hemodiálisis, fue dada de alta con rehabilitación física y seguimiento ambulatorio por los servicios de Nefrología y Medicina Interna. Los familiares de la paciente firmaron el consentimiento informado para la publicación del reporte del caso, durante la estancia de la paciente en UCl.

\section{Discusión}

\section{Epidemiología}

El SHU afecta usualmente a niños entre 18 meses y 4 años $[6,7]$; y aunque es raro después de los 5 años de edad $[8,9]$, también se ha reportado en adultos $[3,10]$. El brote más grande de la infección por STEC en su variante 0104:H4 asociado con SHU, se reportó en Alemania en 2011, el cual invo- lucró 845 casos confirmados de SHU con la afectación de manera inusual de un gran número de adultos (88\%) con predominio del sexo femenino (68\%) [3]. En Estados Unidos, la principal causa de SHU es por STEC, específicamente el serotipo O157:H7, que alcanza el $90 \%$ de los casos $[8,11,12]$. La transmisión se da persona a persona por vía fecal-oral o por la ingestión de comida contaminada $[8,11,13]$. Según su etiología puede ser clasificado como típico, el cual es secundario a una infección, principalmente por STEC, o puede ser atípico debido a una desregulación del complemento, el cual es raro y tiene una incidencia anual $<2$ casos por millón de habitantes $[6,8,14]$.

La importancia de esta patología radica en sus complicaciones, ya que puede causar lesión renal aguda que lleva a requerimiento de terapia de reemplazo renal, la cual puede persistir más allá de la hospitalización, o presentarse falla orgánica múltiple e incluso conducir a la muerte del paciente. Aunque la mayoría de los casos (75\%) tienen una recuperación com-

\begin{tabular}{lccc}
\hline \multicolumn{4}{l}{ Tabla 2. Paraclínicos adicionales realizados a la paciente } \\
\hline Prueba & $\mathbf{3 0 / 0 3}$ & Egreso 05/06 & Valores normales \\
\hline Fibrinógeno (mg/dL) & 218 & - & $200-393$ \\
\hline Bilirrubina total (mg/dL) & 2,47 & 0,36 & $0,1-1,12$ \\
\hline Bilirrubina indirecta (mg/dL) & 1,78 & 0,16 & $0-0,75$ \\
\hline Bilirrubina directa (mg/dL) & 0,68 & 0,2 & $0,1-0,25$ \\
TP (segundos) & 18,1 & 15,5 & 13,53 \\
TPT (segundos) & 30,1 & 30,8 & 29,62 \\
LDH (U/L) & 1.874 & - & $135-214$ \\
Reticulocitos corregidos (\%) & 1,91 & - & $0,5-1,5$ \\
Haptoglobina (mg/dL) & $<10$ & - & $30-250$ \\
\hline
\end{tabular}

TP: tiempo de protrombina; TPT: tiempo parcial de tromboplastina; LDH: deshidrogenasa láctica. 
pleta [11], esta enfermedad presenta una mortalidad del $1 \%$ al $4 \%$ [12], pudiendo ser mayor en los pacientes $>60$ años [10]. Se describe que hasta un tercio de los pacientes quedan con problemas médicos renales, como insuficiencia renal crónica, trastornos neurológicos, hipertensión y proteinuria a largo plazo [12].

En Colombia se han reportado casos de pacientes con diagnóstico de MAT; no obstante, cursan con púrpura trombocitopénica trombótica o con SHU atípico [15], y hasta el momento no se han documentado casos sobre SHU típico en adultos, y en niños en pocas ocasiones. Según la Asociación de Pacientes con Enfermedades del Complemento, tan solo 15 personas se han diagnosticado con SHU en el país [16], por lo que no hay datos suficientes sobre incidencia o prevalencia en nuestra población.

\section{Fisiopatología}

Todas las formas de MAT se caracterizan por la lesión endotelial en la microvasculatura de los órganos; el SHU típico y atípico se caracterizan, en particular, por afectación principalmente del riñón $[8,13]$. El desencadenante de la lesión endotelial puede ser extrínseco, como en el caso de la infección por STEC, por Streptococcus pneumoniae, o por medicamentos, entre otros factores. También puede ser intrínseco, producto de una alteración del complemento, ya sea adquirido o heredado $[7,17]$. La causa del por qué algunos grupos que cursan con la infección por STEC desarrollan SHU y otros no, todavía se desconoce [11]; sin embargo, se ha sugerido una posible predisposición genética que involucre genes asociados con otras formas de MAT, y se han reportado mutaciones en los genes del complemento en pacientes con SHU por STEC $[11,17]$.

En el SHU típico por STEC, la infección se adquiere por la ingestión de la bacteria, la cual una vez llega al intestino, expresa adhesinas que se adhieren al epitelio intestinal, y debido a lisis bacteriana secretan la toxina $[6,11]$. La subunidad $\beta$ de la toxina se adhiere al receptor de globotriaosilceramida (Gb3), el cual es un glicoesfingolípido presente en las superficies de las células, y forman un complejo que es endocitado, dando lugar a vías apoptóticas que causan la muerte celular, e inician cascadas citotóxicas que activan la respuesta inflamatoria $[11,12,18]$. Se ha evidenciado que los riñones son los más susceptibles a estas lesiones, ya que expresan mayores niveles de Gb3, principalmente en las células del endotelio glomerular [14]; igualmente, la expresión de Gb3 en otros órganos, por ejemplo en el sistema nervioso central, explica la sintomatología neurológica que se presenta en algunos pacientes con SHU [11]; de manera alterna, se ha visto que aquellos órganos que no expresan el receptor Gb3 son resistentes a los efectos tóxicos de la bacteria [11].

El daño endotelial ocurre por distintos mecanismos como la citotoxicidad directa, la alteración de la cascada hemostática, el aumento de quimiocinas y la activación de la vía alterna del complemento, haciendo que se activen fenotipos protrombóticos como el factor von Willebrand y las plaquetas, lo cual estimula más producción de células inflamatorias y se promueve la adhesión de leucocitos a las células endoteliales, llevando a daño en los eritrocitos, disminución de las plaquetas por secuestro en la microvasculatura, y compromiso orgánico $[12,18]$. 


\section{Clínica}

El SHU típico, como se mencionó, es una MAT caracterizada por la tríada de anemia hemolítica microangiopática, trombocitopenia e insuficiencia renal aguda $[2,4,12,19]$. Los pacientes que cursan con esta condición, usualmente presentan un cuadro clínico que se caracteriza por diarrea acuosa, dolor abdominal de tipo cólico y fiebre, aproximadamente dos semanas antes de la instauración del síndrome [2,13]. En el transcurso de los días, entre un $50 \%$ a $85 \%$ de los pacientes presentan diarrea sanguinolenta, que es el principal motivo de consulta $[6,8]$. Posteriormente, aparece la insuficiencia renal, secundaria a la lesión endotelial, y se puede presentar oligoanuria hasta en el $70 \%$ de los pacientes [6]; otros pueden presentar hipertensión, hiponatremia, hiperfosfatemia e hipocalemia, aunque en menor porcentaje [6]. De todos los pacientes, solamente el $10 \%$ presentará la triada característica de manera aguda [8].

En menor medida los pacientes pueden presentar alteraciones neurológicas como irritabilidad, confusión, que puede progresar a déficit focal, convulsiones o coma $[11,20]$. Adicionalmente, se ha descrito la presencia de ceguera [2], compromiso a nivel cardiovascular y pancreático $[6,18]$. De los pacientes que presentan SHU asociado a diarrea, más del $60 \%$ requerirá el inicio de terapia de reemplazo renal, y hasta en el $12 \%$ de los pacientes se puede presentar enfermedad renal estadio 5 o la muerte $[8,13]$.

\section{Diagnóstico}

El diagnóstico de SHU típico se debe considerar en los pacientes que cursan con clínica sugestiva de MAT, particularmente cuando al inicio se presenta con cuadro diarreico, aunque se ha visto que incluso en los casos de SHU atípico, los pacientes también pueden tener diarrea; esto resalta la importancia de confirmar la presencia de STEC $[6,11]$. Se deben tomar muestras de materia fecal tanto para coproscópico (en el cual muchas veces se documenta la presencia de sangre oculta en heces y hematíes, sin microorganismos), como para coprocultivo y FilmArray ${ }^{\circledR}$ gastrointestinal (prueba de PCR que identifica 22 patógenos gastrointestinales), que confirma de manera rápida la presencia de STEC $[5,11]$.

Además de solicitar los exámenes en búsqueda de infección por STEC, idealmente se deben evaluar los niveles de ADAMTS13 (A Disintegrin-like and Metalloprotease with Thrombospondin Type 1 Motif 13), prueba de difícil acceso en nuestro medio, cuya función en el organismo es dividir el factor von Willebrand para que este se pueda unir al colágeno en caso de lesión vascular, y realiza el diagnóstico diferencial con púrpura trombocitopenia trombótica si se encuentra menor al 10\% [21] (tabla 3); la cual además, presenta compromiso neurológico predominante [22], y por ende, tiene un tratamiento y pronóstico diferentes [7,23].

La anemia hemolítica microangiopática se caracteriza por la observación en el frotis de sangre periférica de eritrocitos crenados (equinocitos), esquistocitos y esferocitos $[6,8,9]$, siendo los esquistocitos los más frecuentes, los cuales son secundarios al daño mecánico [24]; de igual manera, pueden estar presentes otros signos de hemólisis como haptoglobina disminuida, hiperbilirrubinemia indirecta, elevación de la $\mathrm{LDH}$ y reticulocitosis $[6,9]$.

La trombocitopenia se da por consumo periférico, y se presenta con tiempos de coagulación normal, lo que permite 
Tabla 3. Diagnósticos diferenciales del síndrome hemolítico urémico (SHU) típico

\begin{tabular}{|c|c|}
\hline Patología & Características \\
\hline Púrpura trombocitopénica trombótica & $\begin{array}{l}\text { ADAMTS13 <10\%, predominan síntomas } \\
\text { neurológicos }\end{array}$ \\
\hline Síndrome hemolítico urémico atípico & $\begin{array}{l}\text { Se asocia con alguna condición reconocida que } \\
\text { active el complemento, presencia de anticuerpos } \\
\text { para el factor } \mathrm{H} \text {, características de MAT }\end{array}$ \\
\hline $\begin{array}{l}\text { Síndrome hemolítico urémico por } \\
\text { Streptococcus pneumoniae (SHU-SP) }\end{array}$ & $\begin{array}{l}\text { Asociado a sepsis/meningitis por Streptococcus } \\
\text { pneumoniae }\end{array}$ \\
\hline Coagulación intravascular diseminada (CID) & $\begin{array}{l}\text { Alteración en la coagulación con TPT e INR } \\
\text { alargados, asociada a sepsis }\end{array}$ \\
\hline Síndrome de HELLP (pre/eclampsia) & $\begin{array}{l}\text { Asociado a embarazo, hipertensión, convulsiones. } \\
\text { Presencia de hemólisis, enzimas hepáticas } \\
\text { elevadas y trombocitopenia }\end{array}$ \\
\hline MAT en enfermedades autoinmunes & $\begin{array}{l}\text { Asociada a síndrome antifosfolípido ( } 14 \% \text { ) y lupus } \\
\text { eritematoso sistémico ( } 8 \% \text { a } 15 \% \text { ). Presencia } \\
\text { de anticuerpos antifosfolípidos, antinucleares o } \\
\text { anti-ADN, TPT alargado y compromiso renal o } \\
\text { poliserositis }\end{array}$ \\
\hline Síndrome de Evans & $\begin{array}{l}\text { Trombocitopenia de tipo inmunológica con } \\
\text { anemia hemolítica (Coombs positiva), no se } \\
\text { observan esquistocitos }\end{array}$ \\
\hline MAT inducida por medicamentos & $\begin{array}{l}\text { Asociada a ticlopidina, quinina, tacrolimus, } \\
\text { ciclosporina A, mitomicina C, gemcitabina y } \\
\text { bevacizumab. Presencia de falla hepática }\end{array}$ \\
\hline MAT asociada a VIH & $\begin{array}{l}\text { Se observa en } 0,3 \% \text { de los pacientes con } \mathrm{VIH} \text {, hay } \\
\text { compromiso renal y signos neurológicos focales }\end{array}$ \\
\hline
\end{tabular}

MAT: microangiopatía trombótica; TPT: tiempo de tromboplastina parcial activado; INR: índice internacional normalizado; VIH: virus de la inmunodeficiencia humana.

diferenciarlo de una coagulación intravascular diseminada (CID) [6]. Las manifestaciones clínicas de la trombocitopenia pueden ser epistaxis, equimosis, petequias, sangrado gingival, hematuria, sangrado vaginal o sangrado menstrual abundante, e incluso pueden presentarse hemorragias retinianas y hemoptisis, 0 puede ser completamente asintomática.

Otro de los paraclínicos importantes es el hemograma, en el cual se puede observar leucocitosis, trombocitopenia que puede ser $<50.000 / u L$, sin alteración de la morfología plaquetaria, y anemia con valores de hemoglobina $<8$ g/dL [20,24]; además, la proteína $C$ reactiva puede estar elevada sig- nificativamente, al igual que la urea y la creatinina, y en el uroanálisis se puede evidenciar hematuria y proteinuria. Asimismo, se debe solicitar serología para VIH, virus de la hepatitis B y $C$, y si es pertinente, prueba de embarazo, para descartar otros diagnósticos diferenciales que cursan con complicaciones similares $[6,20,24,25]$ (tabla 3). En la figura 2 se observa un algoritmo para el diagnóstico diferencial de las principales MAT.

\section{Tratamiento}

Realizar una diferenciación entre los distintos tipos de MAT es de vital im- 
portancia, ya que de ello dependerá el enfoque terapéutico $[5,15]$. El pilar del tratamiento se basa en el manejo intrahospitalario de soporte $[23,26]$, que se debe enfocar en la administración de líquidos endovenosos de manera temprana, ya que reduce la tasa de compromiso a nivel del sistema nervioso central $[9,13,17]$ y la necesidad de terapia de reemplazo renal, lo cual aumenta la supervivencia de los pacientes y evita las secuelas a largo plazo [8].

El uso de antibióticos no se recomienda en la infección por STEC, especialmente las quinolonas, ya que no se ha documentado ningún beneficio e in- cluso se ha visto que pueden inducir la liberación y aumento de la toxina Shiga, al causar lesión en la membrana de las bacterias [11,17,27]; además, pueden generar presión selectiva causando alteración en la flora gastrointestinal, y generar complicaciones como la infección por Clostridium difficile [17]. No obstante, en algunos pequeños estudios que no han tenido reproducibilidad, se ha documentado la disminución de complicaciones a nivel neurológico con su uso [13].

La plasmaféresis no está indicada en todos los tipos de MAT, en algunas de las situaciones en las que se recomienda es en la púrpura trombocitopénica

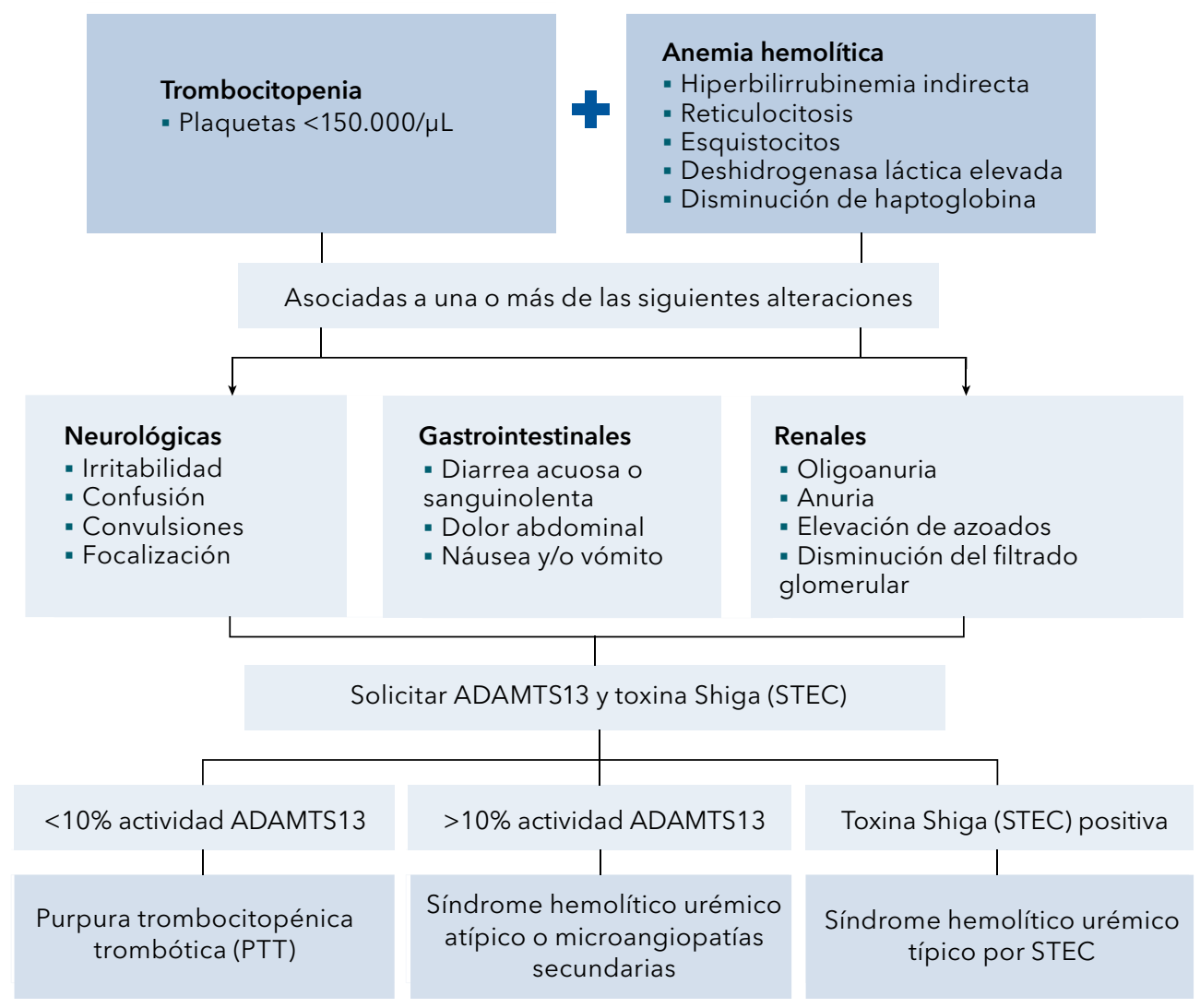

Figura 2. Algoritmo diagnóstico para diferenciar las principales MAT. 
trombótica congénita o en el SHU asociado a neumococo; su indicación en el SHU por STEC no cumple con la evidencia adecuada y dependiendo de la guía, el nivel puede ser 2B o 2C (estudios de cohorte o ensayos clínicos aleatorizados de baja calidad) $[3,11,24]$. Su uso aumentó posterior al impacto que tuvo durante el brote de SHU en Alemania en 2011, donde los pacientes con sintomatología neurológica fueron llevados a recambio plasmático con buenos resultados; sin embargo, no se ha evidenciado aún su beneficio potencial en otras situaciones $[11,13]$.

La transfusión de concentrado de glóbulos rojos está indicada según los criterios habituales, pero la transfusión de plaquetas no es recomendada por muchos expertos, ya que se considera que puede generar microtrombos y exacerbar la enfermedad; no obstante, debido a que no hay suficiente información que soporte o contraindique su beneficio, se recomienda limitar su uso a hemorragia activa y/o procedimientos quirúrgicos invasivos $[11,13,18]$.

Una de las terapias que ha tomado importancia ha sido el uso de eculizumab, un anticuerpo monoclonal anti-C5 [9,17], el cual fue usado en poblaciones pediátricas con síntomas neurológicos, con una adecuada resolución; no obstante, en otras situaciones, como durante el brote de SHU en Alemania, no se evidenció beneficio alguno $[2,11,13,17,26]$.

\section{Conclusiones}

El caso clínico presentado corresponde a una patología infrecuente, ya que durante la revisión de la literatura no se encontraron reportes de casos con SHU en adultos en Colombia; adicionalmente, a nivel mundial continúa siendo una enfermedad de baja incidencia que afecta principalmente a la población pediátrica. Los líquidos endovenosos y la terapia de reemplazo renal son fundamentales en el tratamiento del SHU. Si bien a la paciente del caso descrito se le realizó plasmaféresis, sus beneficios no están demostrados aún por evidencia fuerte. Se debe tener presente esta enfermedad en los pacientes con gastroenteritis aguda con un curso inusual, y que muestren deterioro agudo de la función renal, así como trombocitopenia y anemia hemolítica microangiopática. En estos casos se recomienda realizar las pruebas descritas para documentar la infección por STEC, y así confirmar el diagnóstico y poder brindar el tratamiento oportunamente; de igual manera, se debe tener en cuenta que las MAT tienen características similares, pero tratamientos y pronósticos diferentes, por lo que realizar un diagnóstico preciso es de suma importancia.

\section{Referencias}

1. Arnold DM, Patriquin CJ, Nazy I. Thrombotic microangiopathies: a general approach to diagnosis and management. CMAJ 2017;189:153-159. https://doi.org/10.1503/ cmaj.160142.

2. Cody EM, Dixon BP. Hemolytic uremic syndrome. Pediatr Clin North Am 2019;66:235-246. https://doi.org/10.1016/j.pcl.2018.09.011.

3. Pandey Y, Atwal D, Sasapu A. Diarrheaassociated hemolytic uremic syndrome in adults: Two case reports and review of the literature. Cureus 2019;11:e4435. https://doi. org/10.7759/cureus.4435.

4. Petruzziello-Pellegrini TN, Moslemi-Naeini

M, Marsden PA. New insights into Shiga toxinmediated endothelial dysfunction in hemolytic uremic syndrome. Virulence 2013;4:556-563. https://doi.org/10.4161/viru.26143.

5. Piralla A, Lunghi G, Ardissino G, Girello A, Premoli M, Bava E, et al. FilmArray ${ }^{\top \mathrm{M}} \mathrm{Gl}$ panel performance for the diagnosis of acute gas- 
troenteritis or hemorragic diarrhea. BMC microbiology 2017;17:111. https://doi.org/10.1186/ s12866-017-1018-2.

6. Palacios-Cuesta A, Ordóñez-Sáez O. Cap. 15.3. Síndrome hemolítico urémico. In: Montejo J, García de Lorenzo A, Marco P, Ortiz C, eds. Manual de Medicina Intensiva. 5a ed. Amsterdam, Países Bajos: Elsevier; 2017.

7. Nathanson S, Kwon T, Elmaleh M, Charbit M, Launay EA, Harambat J, et al. Acute neurological involvement in diarrhea-associated hemolytic uremic syndrome. Clin J Am Soc Nephrol 2010;5:1218-1228. https://doi.org/10.2215/ cjn.08921209.

8. McCollough M, Rose E. Genitourinary and renal tract disorders. In: Walls $\mathrm{R}$, Hockberger R, Gausche-Hill M, eds. Rosen's Emergency Medicine: Concepts and Clinical Practice. Amsterdam, Países Bajos: Elsevier; 2018. p. 21632181. https://www.clinicalkey.es/\#!/content/3s2.0-B9780323354790001732.

9. Manoharon A, Schelling JR, Diamond M, Chung-Park M, Madaio M, Sedor JR. Chapter 82 - Immune and inflammatory glomerular diseases. In: Alpern RJ, Moe OW, Caplan M, eds. Seldin and Giebisch's The Kidney. 5th ed. Massachusetts, Estados Unidos: Academic Press; 2013. p. 2763-2816. https://doi.org/10.1016/ B978-0-12-381462-3.00082-3.

10. Ko H, Maymani H, Rojas-Hernandez C. Hemolytic uremic syndrome associated with Escherichia coli 0157:H7 infection in older adults: a case report and review of the literature. J Med Case Rep 2016;10:175. https://doi. org/10.1186/s13256-016-0970-z.

11. Kavanagh D, Raman S, Sheerin NS. Management of hemolytic uremic syndrome. F1000Prime Rep 2014;6:119. https://doi.org/10.12703/ P6-119.

12. Keir LS. Shiga toxin associated hemolytic uremic syndrome. Hematol Oncol Clin North Am 2015;29:525-539. https://doi.org/10.1016/j. hoc.2015.01.007.

13. Bruyand $M$, Mariani-Kurkdjian $P$, Gouali $M$, de Valk H, King LA, Le Hello S, et al. Hemolytic uremic syndrome due to Shiga toxin-producing Escherichia coli infection. Med Mal Infect 2018;48:167-174. https://doi.org/10.1016/j. medmal.2017.09.012.
14. Bowen EE, Coward RJ. Advances in our understanding of the pathogenesis of hemolytic uremic syndromes. Am J Physiol Renal Physiol 2018;314:454-461. https://doi.org/10.1152/ ajprenal.00376.2017

15. Domínguez-Ramírez GA, Blanco-Pertuz PM, Herrera-Rueda GA. Síndrome hemolítico urémico atípico (SHUa): A propósito de un caso de manejo satisfactorio con eculizumab. Acta Med Colomb 2019;44. https://doi.org/10.36104/ amc.2019.1301

16. Universidad Nacional de Colombia. SHU, una enfermedad con 15 casos registrados en Colombia. Bogotá, Colombia: Agencia de Noticias de la Universidad Nacional de Colombia; 2013. Acceso 13 de junio de 2020. Disponible en http://agenciadenoticias.unal.edu.co/detaIle/article/shu-una-enfermedad-con-15-casosregistrados-en-colombia.html.

17. Fakhouri F, Zuber J, Frémeaux-Bacchi V, Loirat C. Haemolytic uraemic syndrome. Lancet 2017;390:681-696. https://doi.org/10.1016/ s0140-6736(17)30062-4.

18. Joseph A, Cointe A, Mariani Kurkdjian $P$, Rafat C, Hertig A. Shiga toxin-associated hemolytic uremic syndrome: A narrative review. Toxins 2020;12:67. https://doi.org/10.3390/ toxins12020067.

19. Obrig TG, Karpman D. Shiga toxin pathogenesis: kidney complications and renal failure. Curr Top Microbiol Immunol 2012;357:105-136. https://doi.org/10.1007/82_2011_172.

20. Scully M, Hunt BJ, Benjamin S, Liesner R, Rose P, Peyvandi F, et al. Guidelines on the diagnosis and management of thrombotic thrombocytopenic purpura and other thrombotic microangiopathies. Br J Haematol 2012;158:323-335. https:// doi.org/10.1111/j.1365-2141.2012.09167.x.

21. Masias C, Cataland SR. The role of ADAMTS 13 testing in the diagnosis and management of thrombotic microangiopathies and thrombosis. Blood 2018;132:903-910. https://doi. org/10.1182/blood-2018-02-791533.

22. Morales-Montoya A. Púrpura trombocitopénica trombótica. Med Int Mex 2019;35:906-911.

23. Joseph A, Rafat $C$, Zafrani L, Mariani-Kurkdjian P, Veyradier A, Hertig A, et al. Early differentiation of Shiga toxin-associated hemolytic uremic syndrome in critically ill adults 
with thrombotic microangiopathy syndromes. Crit Care Med 2018;46:904-911. https://doi. org/10.1097/ccm.0000000000003292.

24. Romero S, Sempere A, Gómez-Seguí I, Román E, Moret A, Jannone $R$, et al. Practice guidelines for the emergency treatment of thrombotic microangiopathy. Med Clin (Barc) 2018;151:123. https://doi.org/10.1016/j.medcli.2018.01.013.

25. Katsuno $T$, Ito $Y$, Kagami S, Kitamura $H$, Maruyama S, Shimizu A, et al. A nationwide cross-sectional analysis of thrombotic microan- giopathy in the Japan Renal Biopsy Registry (J-RBR). Clin Exp Nephrol 2020;24:789-797. https://doi.org/10.1007/s10157-020-01896-7.

26. Mühlen S, Dersch P. Treatment strategies for infections with shiga toxin-producing Escherichia coli. Front Cell Infect Microbiol 2020;10:169 https://doi.org/10.3389/fcimb.2020.00169.

27. Kakoullis L, Papachristodoulou E, Chra P, Panos G. Shiga toxin-induced haemolytic uraemic syndrome and the role of antibiotics: a global overview. J Infect 2019;79:75-94. https://doi. org/10.1016/j.jinf.2019.05.018. 